\title{
Mechano-oxidative coupling by mitochondria induces proinflammatory responses in lung venular capillaries
}

\author{
Hideo Ichimura, ${ }^{1}$ Kaushik Parthasarathi, ${ }^{1}$ Sadiqa Quadri, ${ }^{1}$ Andrew C. Issekutz, ${ }^{2}$ \\ and Jahar Bhattacharya ${ }^{1,3}$ \\ ${ }^{1}$ Lung Biology Laboratory, Department of Physiology and Cellular Biophysics, College of Physicians and Surgeons, \\ St. Luke's-Roosevelt Hospital Center, Columbia University, New York, New York, USA \\ ${ }^{2}$ Department of Pediatrics, Microbiology-Immunology, and Pathology, Dalhousie University, Halifax, Nova Scotia, Canada \\ ${ }^{3}$ Department of Medicine, College of Physicians and Surgeons, St. Luke's-Roosevelt Hospital Center, Columbia University, \\ New York, New York, USA
}

\begin{abstract}
Elevation of lung capillary pressure causes exocytosis of the leukocyte adhesion receptor P-selectin in endothelial cells (ECs), indicating that lung ECs generate a proinflammatory response to pressureinduced stress. To define underlying mechanisms, we followed the EC signaling sequence leading to P-selectin exocytosis through application of real-time, in situ fluorescence microscopy in lung capillaries. Pressure elevation increased the amplitude of cytosolic $\mathrm{Ca}^{2+}$ oscillations that triggered increases in the amplitude of mitochondrial $\mathrm{Ca}^{2+}$ oscillations and in reactive oxygen species (ROS) production. Responses to blockers of the $\mathrm{Ca}^{2+}$ oscillations and of mitochondrial electron transport indicated that the ROS production was $\mathrm{Ca}^{2+}$ dependent and of mitochondrial origin. A new proinflammatory mechanism was revealed in that pressure-induced exocytosis of P-selectin was inhibited by both antioxidants and mitochondrial inhibitors, indicating that the exocytosis was driven by mitochondrial ROS. In this signaling pathway mitochondria coupled pressure-induced $\mathrm{Ca}^{2+}$ oscillations to the production of ROS that in turn acted as diffusible messengers to activate P-selectin exocytosis. These findings implicate mitochondrial mechanisms in the lung's proinflammatory response to pressure elevation and identify mitochondrial ROS as critical to P-selectin exocytosis in lung capillary ECs. J. Clin. Invest. 111:691-699 (2003). doi:10.1172/JCI200317271.
\end{abstract}

\section{Introduction}

The lung's innate immune defense rapidly deploys proinflammatory mechanisms when challenged, leading to the expression of leukocyte adhesion receptors and leukocyte recruitment in pulmonary blood vessels. Although an extensive literature identifies microbes, endotoxins, and cytokines (1-3) as the main challenges, it is now evident that even a modest elevation of lung vascular pressures activates proinflammatory responses in endothelial cells (ECs) of the lung venular capillary (4). This indicates that the lung's immune defense is mechanosensitive and that the pathogenic-

Received for publication October 30, 2002, and accepted in revised form January 10, 2003.

Address correspondence to: Jahar Bhattacharya,

St. Luke's-Roosevelt Hospital Center, 1000 Tenth Avenue,

New York, New York 10019, USA.

Phone: (212) 523-7310; Fax: (212) 523-8005;

E-mail: jb39@columbia.edu.

Conflict of interest: The authors have declared that no conflict of interest exists.

Nonstandard abbreviations used: endothelial cell (EC); cytosolic $\mathrm{Ca}^{2+}\left(\mathrm{Ca}^{2+}\right.$ cyt $)$; mitochondrial $\mathrm{Ca}^{2+}\left(\mathrm{Ca}^{2+}\right.$ mit $)$; reactive oxygen species (ROS); $2^{\prime}, 7^{\prime}$-dichlorodihydrofluorescein diacetate (DCFH-DA); tetramethylrhodamine methyl ester (TMRM); 4,5-diaminofluorescein diacetate (DAF 2DA); $\mathrm{N}^{\mathrm{G}}$-nitro-L-arginine methyl ester (L-NAME); diphenyleneiodonium (DPI); carbonyl cyanide $p$-(trifluoromethoxy)phenylhydrazone (FCCP); $\mathrm{N}$-acetylL-cysteine (NAC); polyethylene glycol-conjugated catalase (PEG-catalase); S-nitroso-N-acetylpenicillamine (SNAP); left atrial pressure (PLA). ity of elevated lung vascular pressures, a condition that predisposes to pulmonary edema (5), could involve proinflammatory events. However, pressure-induced EC signaling mechanisms remain poorly understood.

In the initial stages of inflammation, EC expression of the leukocyte adhesion receptor P-selectin increases leukocyte rolling on the vessel surface (6). In lung, P-selectin expression is pathogenic, as indicated by the protective effects of anti-P-selectin Ab's in several forms of lung injury (7-9). P-selectin is stored in Weibel-Palade bodies in ECs, and its expression results when an increase of the EC cytosolic $\mathrm{Ca}^{2+}\left(\mathrm{Ca}^{2+} \mathrm{cyt}\right)$ induces exocytosis of Weibel-Palade bodies (10). This process is considered similar to that of regulated exocytosis in other secretory cells in which $\mathrm{Ca}^{2+}$ cyt increases trigger vesicle fusion with the plasma membrane and subsequent release of vesicle contents (11). However, it is unclear whether the facilitating effect of $\mathrm{Ca}^{2+}$ on P-selectin exocytosis is direct or whether post- $\mathrm{Ca}^{2+}$ signaling steps are involved.

In this regard, the role of mitochondria requires consideration. There is increasing recognition that mitochondria regulate $\mathrm{Ca}^{2+} \mathrm{cyt}$ by both importing and exporting $\mathrm{Ca}^{2+}$. The negative potential across the mitochondrial inner membrane drives $\mathrm{Ca}^{2+}$ into the mitochondrial matrix across a uniporter, while export is achieved by multiple mechanisms, including an electroneutral $\mathrm{Na}^{+} \backslash \mathrm{Ca}^{2+}$ exchanger (12). Mitochondrial $\mathrm{Ca}^{2+}$ $\left(\mathrm{Ca}^{2+}\right.$ mit) import blunts increases of $\mathrm{Ca}^{2+} \mathrm{cyt}$ that in some cell types, such as pituitary cells (13), diminishes 
secretion. $\mathrm{Ca}^{2+}$ import increases $\mathrm{Ca}^{2+}$ mit and augments mitochondrial production of reactive oxygen species (ROS) (14) that include superoxide and $\mathrm{H}_{2} \mathrm{O}_{2}$. Mitochondrial ROS act on cytosolic targets and are implicated in the activation of transcription factors (15) and in the induction of cytochrome $c$ release, leading to apoptosis (16). However, the role of mitochondria in proinflammatory responses remains unknown.

Here we consider the role of EC mitochondria in responses of the lung venular capillary to pressure stress. A major objective was to determine whether elevation of capillary pressure recruits EC mitochondrial mechanisms as indicated in increases of $\mathrm{Ca}^{2+}$ mit. Our objective was to apply a moderate pressure elevation sufficient to induce a proinflammatory response without overstressing the blood vessels, in order to avoid induction of fluid leakage and vascular injury. Our findings indicate that such a pressure elevation induces P-selectin expression and that this expression is determined by enhanced EC $\mathrm{Ca}^{2+}$ mit oscillations, revealing mitochondria as organelles that couple the mechanical effects of pressure elevation to the capillary's proinflammatory response.

\section{Methods}

Fluorescent dyes and reagents. We used the following fluorescent dyes: fura 2AM, rhod 2AM, MitoTracker Green, 2',7'-dichlorodihydrofluorescein diacetate (DCFH-DA), tetramethylrhodamine methyl ester (TMRM; Molecular Probes Inc., Eugene, Oregon, USA), and 4,5-diaminofluorescein diacetate (DAF 2DA; Calbiochem-Novabiochem Corp., La Jolla, California, USA). Vehicle for dyes was HEPES buffer (150 $\mathrm{mmol} / 1 \mathrm{Na}^{+}, 5 \mathrm{mmol} / 1 \mathrm{~K}^{+}, 1.0 \mathrm{mmol} / \mathrm{l} \mathrm{Ca}^{2+}, 1 \mathrm{mmol} / 1$ $\mathrm{Mg}^{2+}$, and $20 \mathrm{mmol} / \mathrm{l} \mathrm{HEPES}$ at $\mathrm{pH}$ 7.4) containing $4 \%$ dextran $(70 \mathrm{kDa})$ and $1 \%$ FBS. Drugs used were the cell-permeant $\mathrm{Ca}^{2+}$ chelator BAPTA-AM, $\mathrm{N}^{\mathrm{G}}$-nitro-Larginine methyl ester (L-NAME), thapsigargin, rotenone, ebselen, xestospongin $\mathrm{C}$, Trolox, diphenyleneiodonium (DPI), saponin (Calbiochem-Novabiochem Corp.), carbonyl cyanide $p$-(trifluoromethoxy)phenylhydrazone (FCCP), oligomycin, $\mathrm{N}$-acetyl-L-cysteine (NAC), hydrogen peroxide $\left(\mathrm{H}_{2} \mathrm{O}_{2}\right)$, catalase, TNF- $\alpha$, antimycin A, ruthenium red, polyethylene glycol-conjugated catalase (PEG-catalase), arachidonate, allopurinol, and S-nitroso-N-acetylpenicillamine (SNAP) (Sigma-Aldrich, St. Louis, Missouri, USA). FCCP was given with oligomycin $(5 \mu \mathrm{g} / \mathrm{ml})$ to inhibit mitochondrial consumption of cellular ATP (17). Ab's used were mouse anti-rat P-selectin, mAb RP-2 (4), and Alexa Fluor 488-labeled goat anti-mouse IgG (Molecular Probes Inc.).

Real-time digital imaging of the lung venular capillary lung preparation. Using our reported methods $(4,18)$, we pump-perfused lungs from Sprague-Dawley rats (anesthetized with $3.5 \%$ halothane inhalation and $35 \mathrm{mg} / \mathrm{kg}$ intraperitoneal sodium pentobarbital) with autologous blood $(14 \mathrm{ml} / \mathrm{min})$ at $37^{\circ} \mathrm{C}$. Baseline pulmonary artery and left atrial and airway pressures were held constant at 10,5 , and $5 \mathrm{cmH}_{2} \mathrm{O}$, respectively. To increase lung
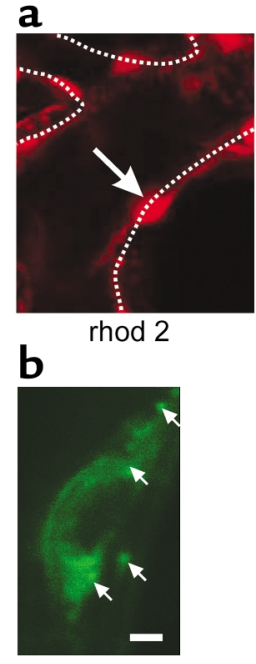

Figure 1

Fluorescence of ECs in situ in a lung venular capillary. (a) Confocal images show a fluorescent EC (arrow). Dotted lines are capillary margins as detected in bright-field images obtained in parallel. Bar $=10 \mu \mathrm{m}$. (b) Single EC imaged at high magnification shows mitochondria as fluorescent clumps (arrows). Bar $=2 \mu \mathrm{m}$. (c) Effect of saponin $(0.01 \%)$ treatment. Capillaries were coloaded with dyes and excited alternately at the wavelengths indicated in parentheses under $\mathrm{Ca}^{2+}$-free conditions. Each point is mean $\pm \mathrm{SE}$ of three determinations. MTG, MitoTracker Green.

capillary pressure, we increased left atrial pressure (PLA) by increasing the height of the venous outflow.

Imaging protocol. We viewed venular capillaries by means of either an image intensifier (Midnight Sun; Imaging Research Inc., St. Catharine's, Ontario, Canada) mounted on a fluorescence microscope (AX-70; Olympus America Inc., Melville, New York, USA) or a laser scanning microscope (LSM 5 Pascal; Carl Zeiss Inc., Thornwood, New York, USA). We identified vessel location and vessel margins under bright-field conditions and obtained images at a focal plane corresponding to maximum capillary diameter at a capture rate of one image every 10 seconds. During imaging, we infused the capillaries with buffer to establish blood-free conditions. Capillary fluorescence was quantified using image analysis software (MCID 5; Imaging Research Inc.).

Using our described protocol (4), we maintained low vascular pressure $\left(\mathrm{PLA}=3 \mathrm{cmH}_{2} \mathrm{O}\right)$ while infusing dyes to load capillary ECs. This protocol prevents dye leakage from the capillary and ensures dye loading specifically in ECs lining the capillary wall. We infused the following dyes through a wedged venous catheter: (a) fura $2 \mathrm{AM}$ (10 $\mu \mathrm{M})$ to quantify $\mathrm{Ca}^{2+}$ cyt by the ratiometric method (18) (alternate excitations at 340 and $380 \mathrm{~nm}$ ); (b) rhod 2AM $(5 \mu \mathrm{M})$ to detect $\mathrm{Ca}^{2+}$ mit (excitation at $550 \mathrm{~nm}$ ); (c) MitoTracker Green $(2.5 \mu \mathrm{M})$ to detect mitochondrial distribution (excitation at $490 \mathrm{~nm}$ ); (d) DCFH-DA $(2.5 \mu \mathrm{M})$ to detect EC ROS production (excitation at $490 \mathrm{~nm}$ ); (e) DAF $2 \mathrm{DA}(3 \mu \mathrm{M})$ to detect EC NO production (excitation at $490 \mathrm{~nm})$; and (f) TMRM $(100 \mathrm{nM})$ to detect mitochondrial potential (excitation at $550 \mathrm{~nm}$ ). All dyes were 
loaded by 20 -minute infusions except TMRM, which was infused for 2 minutes. In fura 2 experiments, the fluorescence ratio was calibrated using fura- $\mathrm{Ca}^{2+}$, which has a $K_{\mathrm{d}}$ of $224 \mathrm{nmol} / \mathrm{l}(18)$. Pseudocolor images reflecting $\mathrm{Ca}^{2+} \mathrm{cyt}$ or fluorescence levels were computer generated. Fura 2 content was detected by single-wavelength excitation at $360 \mathrm{~nm}$. In capillaries loaded with more than one dye, we confirmed the absence of cross-excitation.

To determine EC P-selectin expression, we infused anti-P-selectin mAb (RP-2, $3.5 \mu \mathrm{g} / \mathrm{ml})$ for 4 minutes and then switched the infusion to a fluorescent secondary Ab (Alexa Fluor 488-labeled goat anti-mouse IgG, $10 \mu \mathrm{g} / \mathrm{ml}$ ) for 4 minutes. After a 1-minute flush with buffer, we monitored residual fluorescence, which indicates EC P-selectin expression (4).

Inhibitors were infused for 20 minutes or for other indicated periods through the venous catheter so that infusions reached localized lung regions. In BAPTA-AM $(40 \mu \mathrm{M})$ and thapsigargin experiments, we continuously infused the capillary with $\mathrm{Ca}^{2+}$-free buffer containing $0.5 \mathrm{mM}$ EGTA to maintain external $\mathrm{Ca}^{2+}$-depleted conditions. When loaded in the absence of fluorophores, none of the inhibitors elicited fluorescence in capillaries exposed to the experimental excitation wavelengths (not shown). Ruthenium red is considered by many to be membrane impermeable $(19,20)$; therefore, we minimally permeabilized the capillary membrane using saponin infusion before injecting the inhibitor.

RT-PCR for P-selectin. P-selectin and GAPDH primers were purchased (Life Technologies Inc., Rockville, Maryland, USA). For primer design we used the available P-selectin sequence (GenBank accession number L23088). The sense and antisense primers were, respectively, 5'-GAGCTACACCTGCTCCTGCTAC-3' (nucleotides 555-577) and 5'-GGTTCAC T C CACT GAC CAGAGT C - 3' (nucleotides 962-939). Reverse transcription of total lung RNA and amplification of cDNA were carried out using the one-step SuperScript first-strand synthesis system for RT-PCR. Approximately $1 \mu \mathrm{g}$ of RNA and oligo(dT)30 primer was used for the firststrand cDNA synthesis in a $50-\mathrm{ml}$ reaction mixture. The reverse transcription step was performed at $42^{\circ} \mathrm{C}$ for 50 minutes followed by 15 minutes at $70^{\circ} \mathrm{C}$. PCR was performed for 30 cycles (LABREPCO, Horsham, Pennsylvania, USA) with denaturing at $94^{\circ} \mathrm{C}$ for 1 minute, annealing at $59^{\circ} \mathrm{C}$ for 45 seconds, extension at $72^{\circ} \mathrm{C}$ for 30 seconds, and a final one-step extension at $70^{\circ} \mathrm{C}$ for 15 minutes.

Statistics. Group data are presented as mean \pm SE. Differences between groups were tested by paired $t$ test for two groups and by the ANOVA-NewmanKeuls test for more than two groups. Significance was accepted at $P<0.05$.

\section{Results}

Mitochondria. The $\mathrm{Ca}^{2+}$-binding dye rhod 2 (given as rhod 2AM) de-esterifies intracellularly to cell-impermeant rhod 2 that localizes to the mitochondrial matrix $(21,22)$. This localization was evident by the fluorescence distributions for rhod 2 and MitoTracker Green, which were identical in capillaries coloaded with both dyes (Figure 1a). Viewed at high magnification, mitochondria were labeled as clumps of about $1 \mu \mathrm{m}$ surrounding a nonfluorescent zone representing the nucleus (Figure 1b). To determine cytosolic sequestration, we infused saponin, which permeabilizes EC membranes and releases cytosolic dyes (1). In capillaries coloaded with rhod 2 and the cytosolic marker fura 2, saponin reduced fura 2 fluorescence as expected, although it did not decrease rhod 2 fluorescence (Figure 1c), indicating that rhod 2 was not present in the cytosol.

$\mathrm{Ca}^{2+}$ oscillations. Increasing PLA from 5 to $15 \mathrm{cmH}_{2} \mathrm{O}$ increased capillary diameter from $22.5 \pm 0.6 \mu \mathrm{m}$ to

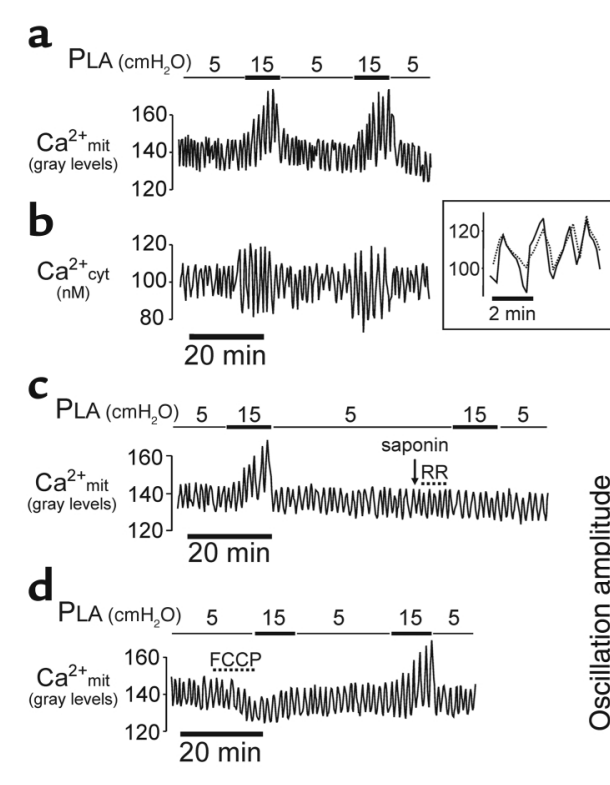

\section{Figure 2}

$\mathrm{Ca}^{2+}$ oscillations in ECs of lung capillaries. (a-f) Traces from single capillaries show responses to PLA elevation under control conditions ( $\mathbf{a}$ and $\mathbf{b}$ ) and in the presence of ruthenium red $(\mathrm{RR}, 10 \mu \mathrm{M})(\mathbf{c}), \mathrm{FCCP}(400 \mathrm{nM})(\mathbf{d})$, xestospongin $\mathrm{C}(\mathrm{XC}, 25 \mu \mathrm{M})$, and arachidonate (AA, $10 \mu \mathrm{M})(\mathbf{e}$ and $\mathbf{f})$ given as indicated. Inset in $\mathbf{b}$ shows determinations of rhod 2 (dotted line) and fura 2 (solid line) fluorescence obtained by alternate excitations at $550 \mathrm{~nm}$ and $340 \mathrm{~nm}$ in a single cell loaded with both dyes. Time scale is expanded to show oscillation synchronicity. Tracing in c was obtained under extracellular $\mathrm{Ca}^{2+}$-free conditions. Inhibitor was given after a 1 -minute infusion of $0.005 \%$ saponin (arrow). In all traces, capillaries were infused with buffer throughout. (g) Group data show oscillation amplitudes in untreated control capillaries $(C T)$ and in capillaries given ruthenium red $(10 \mu \mathrm{M}), \mathrm{FCCP}(\mathrm{FC}, 400 \mathrm{nM})$, xestospongin $\mathrm{C}$ $(25 \mu \mathrm{M})$, and thapsigargin $(\mathrm{TG}, 2 \mu \mathrm{M}) .{ }^{*} P<0.05$. Mean $\pm \mathrm{SE}, n=3$ for each bar. 

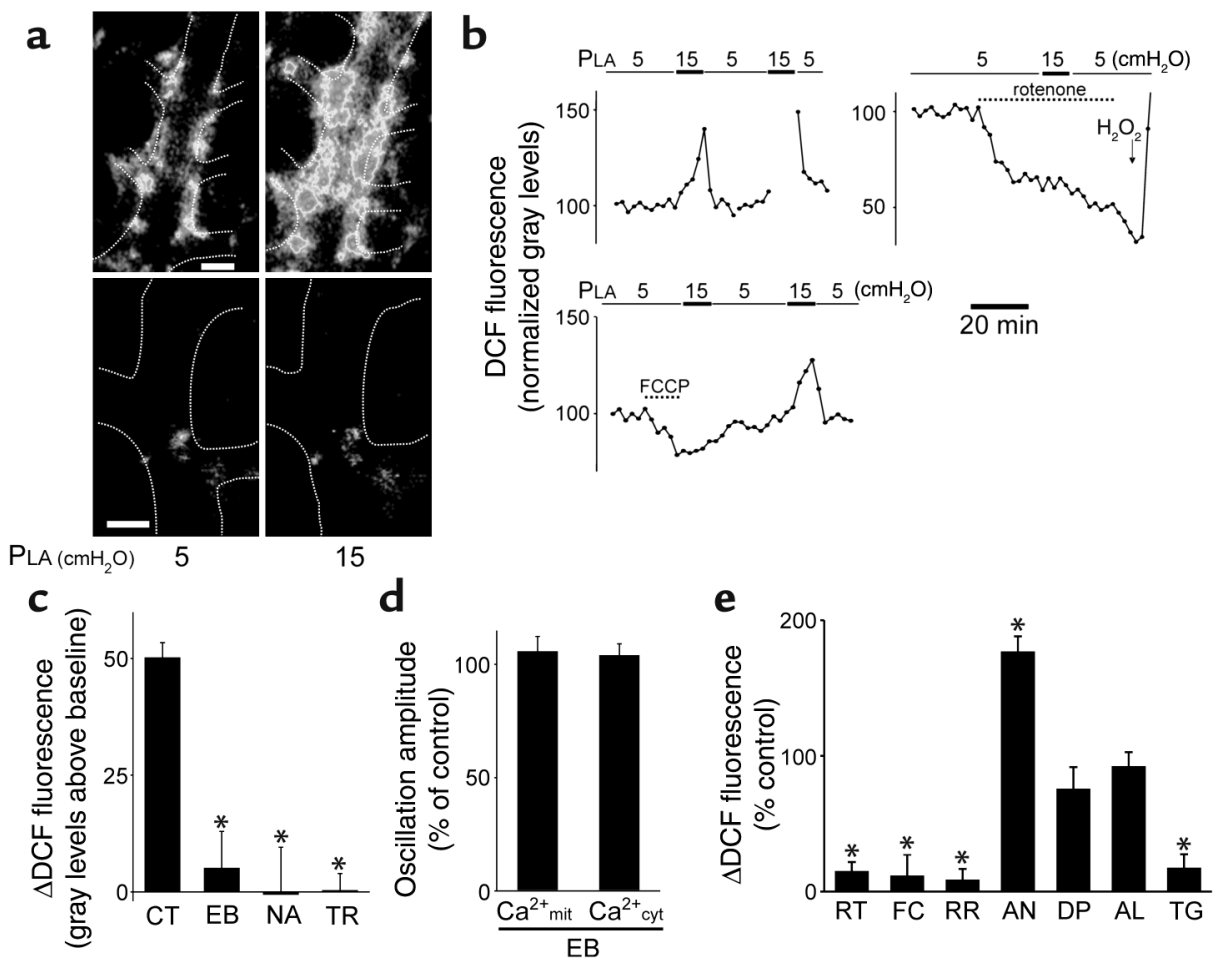

\section{Figure 3}

Detection of ROS in lung venular capillaries. (a) Images show PLA-induced DCF fluorescence in capillaries following infusion of either vehicle (upper images) or rotenone (lower images). Higher grey levels indicate higher fluorescence intensity. Bar $=10 \mu \mathrm{m} ; n=4$. (b) Tracings show time course of responses in single capillaries. Gap in tracing (upper left) shows interruption of imaging during the second pressure challenge. Rotenone $(1 \mu \mathrm{M})$ and $\mathrm{H}_{2} \mathrm{O}_{2}(100 \mu \mathrm{M}$; upper right) and FCCP $(400 \mathrm{nM}$; lower left) were infused in capillaries. (c) Pressure-induced increase of DCF fluorescence in capillaries treated with NAC (NA, $10 \mathrm{mM}$ ), ebselen (EB, $100 \mu \mathrm{M})$, and Trolox (TR, $2 \mathrm{mM})$. ${ }^{*} P<0.05$ compared with untreated control. Mean $\pm \mathrm{SE} ; n=3$ for each group. (d) Pressure-induced responses of $\mathrm{Ca}^{2+}$ mit and $\mathrm{Ca}^{2+} \mathrm{cyt}$ in capillaries treated with ebselen $(100 \mu \mathrm{M})$. Control responses were obtained as paired determinations in the pretreatment period. Mean $\pm \mathrm{SE} ; n=3$ for each group. (e) Group data show pressure-induced responses in capillaries treated with rotenone $(\mathrm{RT}, 1 \mu \mathrm{M}), \mathrm{FCCP}(\mathrm{FC}, 400 \mathrm{nM})$, ruthenium red (RR, $10 \mu \mathrm{M})$, antimycin $A(A N, 1 \mu \mathrm{g} / \mathrm{ml})$, diphenyleneiodonium (DP, $10 \mu \mathrm{M})$, allopurinol (AL, $100 \mu \mathrm{M})$, and thapsigargin (TG, $2 \mu \mathrm{M})$. Control responses were obtained as paired determinations in the pretreatment period. Ruthenium red was given after $0.005 \%$ saponin infusion for 1 minute. *Different from control at $P<0.05$. Data are presented as mean $\pm \mathrm{SE} ; n=3$ for each group.

$27.4 \pm 0.6 \mu \mathrm{m}(P<0.05)$. Concomitantly, the amplitudes of $\mathrm{Ca}^{2+} \mathrm{cyt}$ and $\mathrm{Ca}^{2+}$ mit oscillations increased twofold (Figure 2, a and b). However, there were no increases in mean $\mathrm{Ca}^{2+}$ cyt or in oscillation frequency. Reducing pressure returned oscillation amplitude to baseline for both $\mathrm{Ca}^{2+} \mathrm{cyt}$ and $\mathrm{Ca}^{2+}$ mit; moreover, the responses could be repeated in the same capillary (Figure 2 , a and $b$ ), thereby ruling out cell injury as a factor in these responses. Simultaneous determinations in the same cell indicated that the $\mathrm{Ca}^{2+}$ cyt and $\mathrm{Ca}^{2+}$ mit oscillations were synchronous (Figure $2 \mathrm{~b}$, inset), consistent with the notion that increases of $\mathrm{Ca}^{2+}$ mit rapidly follow those of $\mathrm{Ca}^{2+} \mathrm{cyt}(21,22)$.

To detect the mechanistic sequence of these $\mathrm{Ca}^{2+}$ responses, we used ruthenium red and FCCP, which block $\mathrm{Ca}^{2+}$ mit uptake by inhibiting the mitochondrial uniporter $(21,23)$ and by abrogating the mitochondrial potential difference, respectively $(24,25)$. Since ruthenium red may be membrane impermeable in ECs $(19,20)$, we lightly permeabilized the capillary membrane by infusing saponin in $\mathrm{Ca}^{2+}$-free buffer prior to infusing the inhibitor. This saponin treatment itself did not modify the control response to PLA elevation (not shown), indicating that the response was sustained under $\mathrm{Ca}^{2+}$-free conditions. Both ruthenium red and FCCP blocked the $\mathrm{Ca}^{2+}$ mit, but not the $\mathrm{Ca}^{2+} \mathrm{cyt}$ response (Figure 2, c, d, and g). Since FCCP is a protonophore and may induce nonspecific toxicity, we infused FCCP at a concentration of $400 \mathrm{nM}$, rather than at the higher concentrations of $2 \mu \mathrm{M}$ that was used for Hela and hepatoma cells $(24,26)$. At this concentration, the pressure effect could be rescued by washing FCCP from the capillary (Figure 2d), indicating that the inhibition was reversible and therefore probably not attributable to a toxic effect.

To determine the role of $\mathrm{Ca}^{2+}$ released from endoplasmic stores in these responses, we infused xestospongin $\mathrm{C}$, which blocks release of stored $\mathrm{Ca}^{2+}$ by inhibiting inositol 1,4,5-triphosphate receptors (27) (Figure 2, e-g), and thapsigargin, which blocks release of stored $\mathrm{Ca}^{2+}$ by inhibiting $\mathrm{Ca}^{2+}$ uptake in the ER (28) (Figure 2g). Both inhibitors blocked the $\mathrm{Ca}^{2+}$ mit and $\mathrm{Ca}^{2+}$ cyt responses, indicating that release of stored $\mathrm{Ca}^{2+}$ was activated during pressure elevation. To determine whether $\mathrm{Ca}^{2+} \mathrm{cyt}$ alone determined the mitochondrial response, we infused arachidonate, 
which increases $\mathrm{Ca}^{2+} \mathrm{cyt}$ by causing direct entry of external $\mathrm{Ca}^{2+}$ but causes neither release of $\mathrm{Ca}^{2+}$ from stores nor increase of $\mathrm{Ca}^{2+}$ mit (29). Following xestospongin $\mathrm{C}$ treatment (thus with store release inhibited), arachidonate given together with PLA elevation increased $\mathrm{Ca}^{2+} \mathrm{cyt}$ but not $\mathrm{Ca}^{2+}$ mit (Figure 2, e and f). Taken together, these results indicate that increase of $\mathrm{Ca}^{2+}$ cyt alone was not sufficient to increase $\mathrm{Ca}^{2+}$ mit, and that store release was required for the mitochondrial response.

$R O S$. To determine ROS production, we infused capillaries with membrane-permeant DCFH-DA, which deesterifies intracellularly to DCFH, the substrate that ROS oxidize to fluorescent DCF (26). DCF fluorescence was evident in the capillary wall and not in the pericapillary space, indicating that DCFH-DA was specifically loaded in ECs (Figure 3a). DCFH-loaded capillaries imaged at 20-second intervals evoked relatively low fluorescence at baseline but became intensely fluorescent upon increasing PLA to $15 \mathrm{cmH}_{2} \mathrm{O}$ (Figure 3a) within 1 minute (Figure $3 \mathrm{~b}$ ). Capillary imaging in the final minute of the pressure elevation period also revealed an identical increase of DCF fluorescence (Figure 3b), indicating that imaging-induced artifacts were not present. In the postchallenge period, the fluorescence decreased rapidly (Figure 3b), indicating absence of cell injury.

The pressure-induced increase of DCF fluorescence was almost completely blocked by antioxidants: ebselen, a glutathione peroxidase mimetic; NAC, which enhances the scavenging of ROS by increasing intracellular pools of reduced glutathione; and Trolox, a water-soluble vitamin E derivative (Figure 3c). This blocking of DCF fluorescence affirms that the response was attributable to ROS. However, ebselen did not block the pressureinduced increases of $\mathrm{Ca}^{2+}$ mit or $\mathrm{Ca}^{2+}$ cyt (Figure 3d), therefore these $\mathrm{Ca}^{2+}$ responses were not ROS-induced.

The mitochondrial inhibitors rotenone (which blocks at complex I) and FCCP each reduced DCF fluorescence at baseline (images shown for rotenone effect only in Figure 3a) and also markedly abolished the pressure-induced fluorescence increase (Figure 3, a, b, and e). Ruthenium red given after saponin as described above also blocked the fluorescence increase (Figure 3e). The FCCP-induced inhibition was reversed by washing out the capillary (Figure 3b), indicating absence of inhibitor toxicity. Further, antimycin A, which increases superoxide formation at complex III by inhibiting the conversion of ubisemiquinone to ubiquinone (30), markedly enhanced DCF fluorescence during pressure elevation (Figure 3e). DPI, which blocks NADPH oxidase, and allopurinol, which blocks xanthine oxidase, each failed to abrogate the pressure-induced ROS response (Figure 3e). Taken together, these findings indicated that the induced ROS were of mitochondrial origin. Furthermore, the inhibitions by ruthenium red and FCCP, and the ability of thapsigargin to block both $\mathrm{Ca}^{2+}$ mit oscillations (Figure 2, $\mathrm{c}$ and g) and the DCF fluorescence response (Figure 3e), indicated that the ROS induction was dependent on $\mathrm{Ca}^{2+}$ mit.

We carried out several experiments to affirm the adequacy of the DCF method for ROS detection. Potentially, light-induced effects on DCFH (31) or DCF (32) during imaging could induce fluorescence-enhancing artifacts. However, continuous DCFH-DA infusion imaging at 20second intervals gave stable fluorescence for more than 20 minutes (Figure $3 \mathrm{~b}$ and Figure $4 \mathrm{a}$ ), thereby ruling out light-induced artifacts. Discontinuing DCFH-DA infusion decreased fluorescence to background levels with a half-time of 5 minutes (Figure 4a), consistent with the notion that the cell continually extrudes DCF (33). A bolus of $\mathrm{H}_{2} \mathrm{O}_{2}$ rapidly increased DCF fluorescence, which returned to baseline after washout of the bolus (Figure $4 \mathrm{~b})$, indicating that the method sensitively detected oxidant-induced fluorescence transients. The facts that these fluorescence transients occurred in a concentrationdependent manner (Figure 4c) and that inhibitor-induced blockade of the response was rescued by $\mathrm{H}_{2} \mathrm{O}_{2}$ infusion (shown for rotenone alone in Figure 3a) excluded nonspecific effects as the cause of the inhibitory responses.

To determine the role of $\mathrm{H}_{2} \mathrm{O}_{2}$, we infused membraneimpermeable catalase, which degrades extracellular $\mathrm{H}_{2} \mathrm{O}_{2}$ to molecular oxygen and water. Catalase infusion did not block the pressure-induced DCF response,
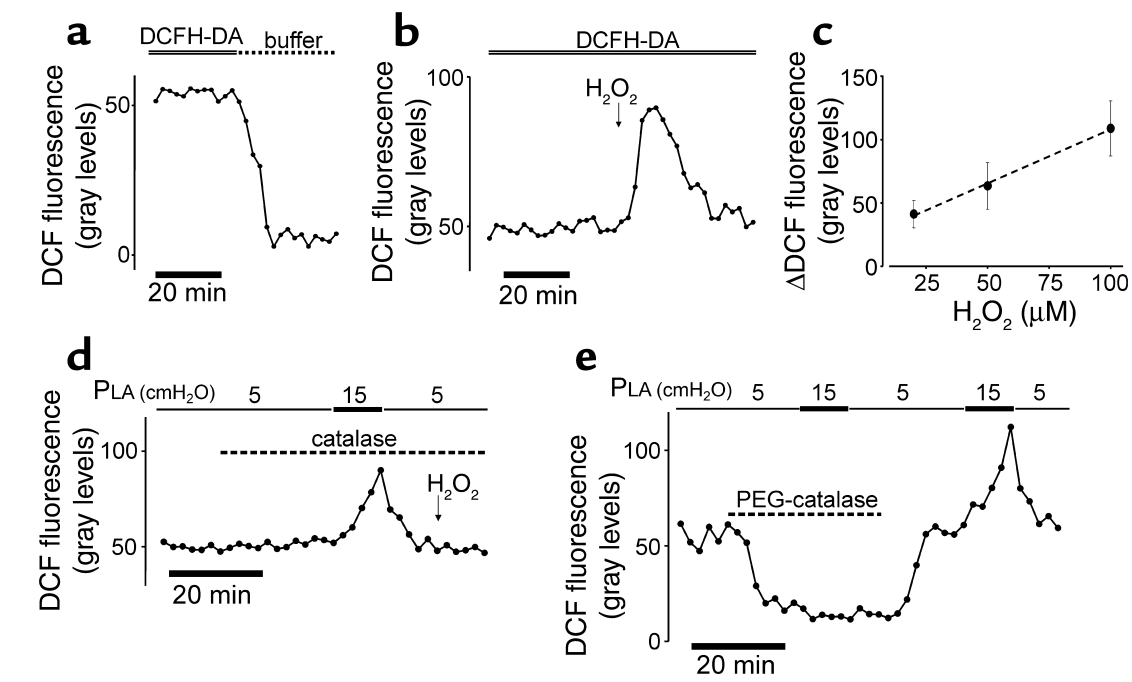

\section{Figure 4}

Induced ROS transients in lung venular capillaries. DCFH-DA $(2.5 \mu \mathrm{M})$ was infused for the indicated durations. DCF fluorescence transients are shown following cessation of DCFH-DA infusion (a) and after a single bolus of $\mathrm{H}_{2} \mathrm{O}_{2}(20 \mu \mathrm{M})(\mathbf{b})$. Peak fluorescence increases above baseline are plotted against bolus concentration (c) (line drawn by linear regression; slope at $P<0.05 ; n=5$ for each point; mean $\pm \mathrm{SE})$. (d) Effect of catalase $(1,000 \mathrm{U} / \mathrm{ml})$ on DCF fluorescence. $\mathrm{H}_{2} \mathrm{O}_{2}(100 \mu \mathrm{M})$ was given as indicated. (e) Trace shows DCF fluorescence in the presence or absence of PEG-catalase $(100 \mathrm{U} / \mathrm{ml})$. Replicated three times. 

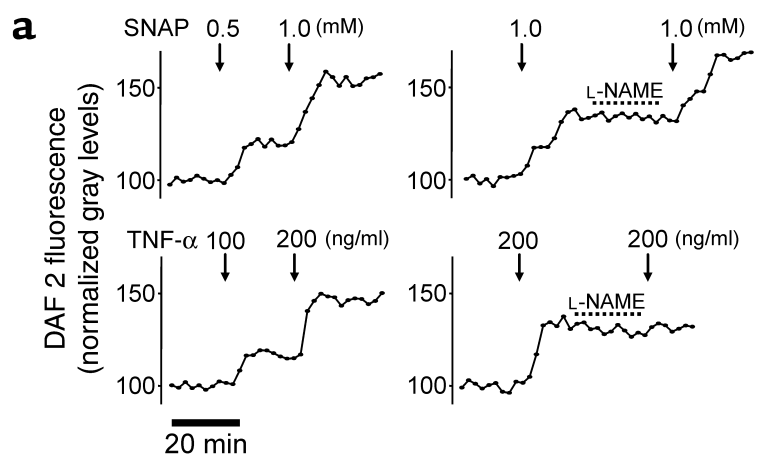

b
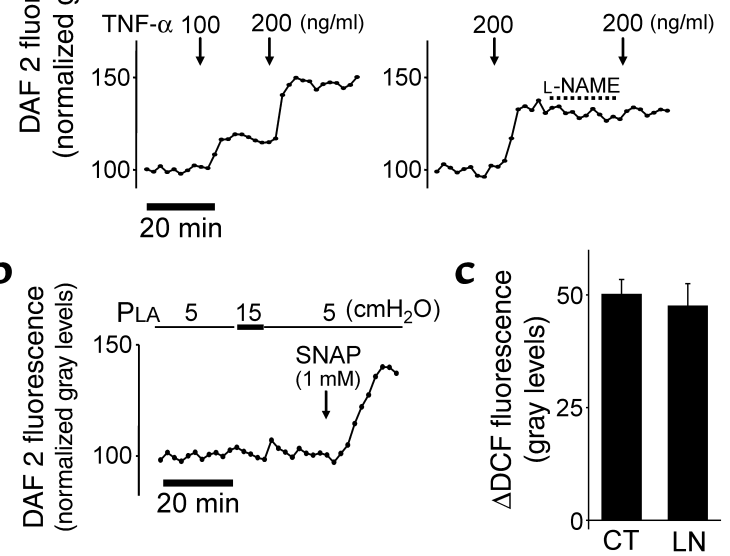

Figure 5

Endothelial NO detection in lung capillaries. (a) Single capillary traces show responses of DAF 2 fluorescence induced by the NO donor SNAP (upper traces) and TNF- $\alpha$ (lower traces) in the absence and presence of the NO synthase inhibitor L-NAME $(10 \mu \mathrm{M})$. (b) Single capillary trace shows effect of PLA elevation. (c) Group data show responses of DCF fluorescence to PLA elevation in untreated $(C T)$ and L-NAME-treated ( $\mathrm{LN} ; 10 \mu \mathrm{M}$ ) capillaries (mean $\pm \mathrm{SE} ; n=3$ for each group).

although as expected, it blocked the fluorescence increase in response to exogenous $\mathrm{H}_{2} \mathrm{O}_{2}$ (Figure 4d). In an experiment not shown, we injected ebselen by micropuncture of an alveolus adjacent to a DCF-loaded capillary. This intra-alveolar ebselen injection failed to modify the pressure-induced DCF fluorescence response in the capillary. These findings indicated that the fluorescence increase in ECs did not result from entry of diffusible ROS from exogenous sources.

To determine the role of intracellular $\mathrm{H}_{2} \mathrm{O}_{2}$, we infused the membrane-permeable form of catalase, PEG-catalase, which rapidly decreased DCF fluorescence at baseline (Figure 4e), indicating quick intracellular entry of the enzyme. In capillaries treated with PEG-catalase, the pressure-induced ROS response was completely blocked (Figure 4e). Cessation of PEG-catalase infusion rapidly restored both baseline levels of DCF fluorescence and the control ROS response to pressure (Figure 4e), indicating that the inhibitor did not cause loss of fluorophore. These findings indicate that $\mathrm{H}_{2} \mathrm{O}_{2}$ was the major constituent of the ROS indicated by DCF fluorescence.

NO. Since NO could potentially oxidize DCFH (34), we determined NO production using the membrane-permeable dye DAF 2DA, which converts to fluorescent DAF $2 \mathrm{~T}$ (triazolofluorescein) in the presence of $\mathrm{NO}$ (35). A potential artifact attributable to photoactivation of DAF 2 (36) was excluded since fluorescence was stable at baseline and did not progressively increase (Figure 5a). Sensitivity of NO detection was indicated in that in DAF 2loaded capillaries, dose-dependent fluorescence increases occurred in response to both the NO donor SNAP and the NO inducer TNF- $\alpha$ (37) (Figure 5a). As expected, the NO synthase inhibitor L-NAME blocked the response to TNF- $\alpha$ but not to SNAP (Figure 5a). However, pressure elevation did not increase DAF 2 fluorescence (Figure $5 \mathrm{~b}$ ), indicating that the pressure challenge did not evoke an NO response. This negative response was not due to poor dye loading, since SNAP infusion rescued the fluorescence increase (Figure 5b). Moreover, L-NAME did not affect the pressure-induced increase of DCF fluorescence (Figure 5c). Taken together, these findings ruled out NO as a factor in the DCF responses.

Mitochondrial potential. In some cell types, ROS-induced damage to mitochondrial membranes causes mitochondrial depolarization that is detected by the decrease of mitochondrial fluorescence of the voltage-sensitive dye TMRM (24). Since cytosolic trapping of TMRM could lead to erroneous assessment of mitochondrial fluorescence, we permeabilized capillaries with saponin to release cytosolic dye. Cell permeabilization also abrogates the potential difference across cell membranes; therefore it is likely that saponin treatment abolished EC membrane potentials that could potentially determine TMRM uptake in mitochondria $(38,39)$. In capillaries thus treated, TMRM fluorescence was sustained at steady levels at both baseline and elevated PLA, while the mitochondrial depolarizer FCCP induced the expected loss of fluorescence (24) (Figure 6). These findings indicate that the present levels of ROS production did not cause detectable mitochondrial damage.

$P$-selectin. To detect P-selectin expression, we determined the immunofluorescence of mAb RP-2. As we have shown previously (4), pressure elevation increased P-selectin expression (Figure 7a) and this effect was inhibited by the cell $\mathrm{Ca}^{2+}$ chelator BAPTA-AM (not shown). Our new findings were that P-selectin expression was completely inhibited by both the mitochondrial inhibitors, rotenone and FCCP (Figure 7, $a$ and b). In the presence of these inhibitors, $\mathrm{P}$-selectin expression could be rescued by exposing the capillaries to $\mathrm{H}_{2} \mathrm{O}_{2}$, indicating that the inhibitory effects did not result from blockade of exocytosis by nonspecific effects such as ATP depletion (Figure 7b, inset). P-selectin expression

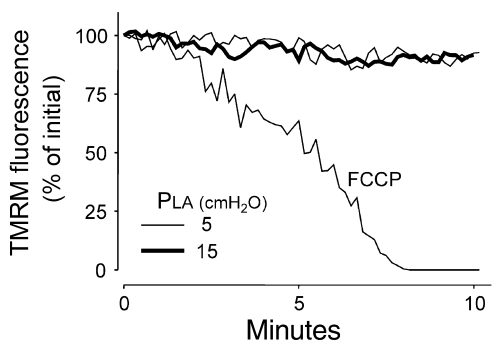

\section{Figure 6}

Effect of pressure elevation on mitochondrial potential. Tracings are quantifications of TMRM fluorescence from a single capillary. Capillaries were first loaded with TMRM, then given saponin (0.01\%) infusion to permeabilize EC membranes. PLA was maintained at $5 \mathrm{cmH}_{2} \mathrm{O}$ (light line) or $15 \mathrm{~cm} \mathrm{H}_{2} \mathrm{O}$ (bold line). Response to FCCP $(2 \mu \mathrm{M})$ was obtained at PLA of $5 \mathrm{~cm} \mathrm{H}_{2} \mathrm{O}$. Traces represent data replicated in four capillaries. 

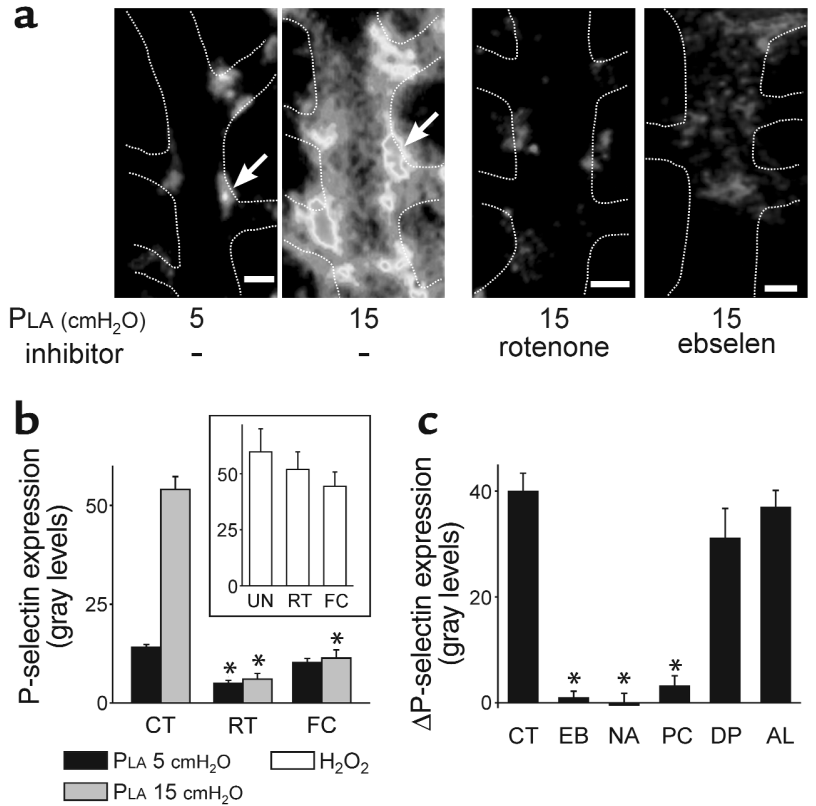

\section{Figure 7}

P-selectin expression in lung capillaries. Each inhibitor was infused for 20 minutes prior to imaging. (a) Images of single capillaries show immunofluorescence of P-selectin at varying PLA in the absence (left pair) and presence (right pair) of the indicated inhibitors. Arrows pointing to the same EC in the same capillary show fluorescence increase. Images show fluorescence intensity as gray levels. Bar $=10 \mu \mathrm{m}$. (b) Responses to the indicated PLA are shown for untreated capillaries and capillaries given mitochondrial blockers $(1 \mu \mathrm{M}$ rotenone or $400 \mathrm{nM}$ FCCP). Responses are after 10 minutes of pressure elevation. ${ }^{*} P<0.05$ compared with control; mean $\pm \mathrm{SE} ; n=3$ for each group. Inset shows responses to a 5-minute $\mathrm{H}_{2} \mathrm{O}_{2}$ infusion $(100 \mu \mathrm{M})$ in the absence of any agents (UN) and in the presence of rotenone and FCCP $(2 \mu \mathrm{M})$. (c) Responses to PLA of $15 \mathrm{cmH}_{2} \mathrm{O}$ shown as difference from baseline in untreated capillaries $(C T)$ and in capillaries treated with ebselen (EB, $100 \mu \mathrm{M})$, NAC (NA, $10 \mathrm{mM}$ ), PEG-catalase (PC, $100 \mathrm{U} / \mathrm{ml}$ ), diphenyleneiodonium (DP, $10 \mu \mathrm{M})$, and allopurinol ( $\mathrm{AL}, 100 \mu \mathrm{M})$. ${ }^{*} P<0.05$ compared with control; mean $\pm \mathrm{SE} ; n=3$ for each group.

was also inhibited by ebselen (Figure 7, a and c), NAC, and PEG-catalase, but not by DPI and allopurinol (Figure 7c). Taken together, these findings indicate that the exocytosis of $\mathrm{P}$-selectin was induced by ROS, in particular $\mathrm{H}_{2} \mathrm{O}_{2}$, of mitochondrial origin.

In lungs held for 2 hours at a PLA of either 5 or 15 $\mathrm{cmH}_{2} \mathrm{O}$, P-selectin mRNA levels were similar (Figure 8). Hence, transcriptional mechanisms did not play a role in the P-selectin responses described here.

\section{Discussion}

Our findings indicate that a modest pressure challenge in lung venular capillaries induced EC mitochondrial responses. These were evident in that $\mathrm{Ca}^{2+}$ mit as reflected in rhod 2 fluorescence, and ROS production as indicated by DCF fluorescence were inhibited by rotenone, ruthenium red, and FCCP. Further, ROS production was augmented by antimycin $\mathrm{A}$. In conjunction, we used fura 2 and in situ immunofluorescence of mAb RP-2, as described previously (4), to determine, respectively, cytosolic second messenger responses in terms of changes in $\mathrm{Ca}^{2+} \mathrm{cyt}$ and initiation of the inflammatory response in terms of $\mathrm{P}$-selectin expression. Our principal findings were that pressure increase augmented oscillation amplitudes for both $\mathrm{Ca}^{2+} \mathrm{cyt}$ and $\mathrm{Ca}^{2+}$ mit, mitochondrial ROS production, and P-selectin expression.

Inhibiting the increase of oscillation amplitude for $\mathrm{Ca}^{2+} \mathrm{cyt}$ inhibited the like response for $\mathrm{Ca}^{2+} \mathrm{mit}$, but not vice versa, indicating that $\mathrm{Ca}^{2+}$ cyt determined the $\mathrm{Ca}^{2+}$ mit respose. Inhibiting the oscillation response for $\mathrm{Ca}^{2+}$ mit inhibited mitochondrial ROS production, indicating that $\mathrm{Ca}^{2+}$ mit directly determined ROS production. However, inhibiting mitochondrial ROS production had no effect on $\mathrm{Ca}^{2+}$ cyt and $\mathrm{Ca}^{2+}$ mit oscillations, ruling out a role for mitochondrial ROS as a determinant of these $\mathrm{Ca}^{2+}$ responses. Inhibiting mitochondrial ROS inhibited P-selectin expression, demonstrating that mitochondrial ROS induced P-selectin expression. Therefore in these ECs, pressure elevation induced a signaling sequence in which an increase of $\mathrm{Ca}^{2+}$ cyt oscillations increased $\mathrm{Ca}^{2+}$ mit oscillations that augmented mitochondrial ROS production. In turn, the ROS caused exocytosis of P-selectin. To identify the specific ROS involved, we infused PEG-catalase, which loads cultured cells after prolonged exposure (40). However, PEG-catalase entered ECs within minutes, as indicated by reversible decreases in DCF fluorescence (Figure 4e). Importantly, PEG-catalase blocked both the pressure-induced DCF fluorescence and P-selectin exocytosis, indicating that $\mathrm{H}_{2} \mathrm{O}_{2}$ was the major ROS that determined these responses. These findings provide a novel view of EC mitochondria in these capillaries as couplers of the pressure-induced $\mathrm{Ca}^{2+} \mathrm{cyt}$ response to proinflammatory exocytosis of P-selectin.

Methodological considerations. Key to these interpretations were our determinations of $\mathrm{Ca}^{2+}$ mit and ROS. The $\mathrm{Ca}^{2+}$ mit determination was based on cationic rhod 2 that localizes to the anionic mitochondrial matrix $(21,22)$. Cytosolic presence of the dye could reduce specificity for the $\mathrm{Ca}^{2+}$ mit determination. However, permeabilization studies with saponin indicated that while cytosolic fura 2 was quickly released from the cell, rhod 2 fluorescence held steady, indicating that rhod 2 was not compartmentalized in the cytosol. Further, ruthenium red and FCCP blocked the pressure-induced responses for rhod 2 but not those for fura 2 , indicating that $\mathrm{Ca}^{2+} \mathrm{cyt}$ did not interfere with the $\mathrm{Ca}^{2+}$ mit determination.

DCF fluorescence may result from light-induced photo-oxidation during image acquisition $(31,32)$.

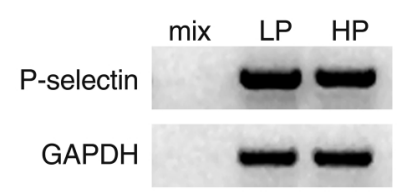

Figure 8

P-selectin mRNA expression in lung. Gels show RT-PCR products for P-selectin mRNA in lungs held for 2 hours at PLA of $5 \mathrm{cmH}_{2} \mathrm{O}(\mathrm{LP})$ or $15 \mathrm{cmH}_{2} \mathrm{O}(\mathrm{HP})$. Controls are shown for PCR buffer (mix) and GAPDH. Replicated three times. 
Photo-activated release of a free radical from DCF may form superoxide on being oxidized by oxygen back to the parent dye (32). We ruled out these potential artifacts since baseline fluorescence did not increase progressively, as would be the case if light-induced DCFH oxidation occurred continuously. Further, repeatable fluorescence transients could be evoked by bolus injections of $\mathrm{H}_{2} \mathrm{O}_{2}$ in a concentration-dependent manner. Reestablishment of the fluorescence baseline after challenge attested to the robustness of our DCF protocol. Since pressure elevation increased capillary diameter by $23 \%$, the resulting effects were likely attributable to EC stretch. Cell stretch in vitro induces ROS and NO through activation of NADPH oxidase and NO synthase, respectively (41-43). However, the present responses were different in that mitochondrial blockers almost entirely abolished the response, while neither DPI nor allopurinol had any effect. As we discuss in Results, our findings with DAF 2 and extravascular catalase and ebselen ruled out respective roles for $\mathrm{NO}$ and for extracellular $\mathrm{H}_{2} \mathrm{O}_{2}$. Taken together, these findings indicate that the DCF fluorescence was attributable to mitochondrial ROS.

Mitochondria and proinflammatory signaling. Mitochondrial ROS production may lead to cell death, as for example in the excitotoxic response in motor neurons (44) and in mitochondrial permeability transition (14). However, to the extent that we could determine, the present levels of ROS were not toxic to mitochondria. We determined TMRM fluorescence, which accumulates in mitochondria in a potential-dependent manner $(24,39)$. FCCP, which abrogates the mitochondrial potential, caused the expected rapid loss of TMRM fluorescence $(24,39)$. However, pressure elevation did not affect fluorescence, indicating that no detectable loss of mitochondrial potential occurred in response to pressure stress. Furthermore, not only was the fluorescence of intracellular indicators such as fura 2 sustained at stable levels both before and after pressure elevation, but both mitochondrial and $\mathrm{Ca}^{2+}$ cyt responses to pressure could be repeated in the same capillary. We interpret that no cell or mitochondrial toxicity occurred in these capillaries and that mitochondrial ROS, in particular $\mathrm{H}_{2} \mathrm{O}_{2}$, played the role of signaling messengers.

Unlike most forms of regulated exocytosis in which $\mathrm{Ca}^{2+}$ cyt plays a direct role in instituting vesicle fusion (45), here the $\mathrm{Ca}^{2+} \mathrm{cyt}$ role was indirect in that a $\mathrm{Ca}^{2+} \mathrm{cyt}-$ induced $\mathrm{Ca}^{2+}$ mit increase was critical to P-selectin expression. However, the increase in $\mathrm{Ca}^{2+}$ cyt alone was not sufficient for this response, since $\mathrm{Ca}^{2+} \mathrm{cyt}$ increases induced by arachidonate failed to increase $\mathrm{Ca}^{2+}$ mit in the presence of pressure increase, provided store release was blocked. This result, together with the inhibitory effects of thapsigargin and xestospongin $\mathrm{C}$, indicates that release of stored $\mathrm{Ca}^{2+}$ was critical in increasing $\mathrm{Ca}^{2+}$ mit $(29,46)$.

Inhibition by ruthenium red indicated that $\mathrm{Ca}^{2+} \mathrm{mit}$ uptake determined ROS production. The inhibitory effect of FCCP is consistent with the view that loss of the mitochondrial proton gradient blocks ROS generation $(25,44,47,48)$, although this inhibition is not universal to all cell types $(49,50)$. The mechanism underlying $\mathrm{Ca}^{2+}$ mit-induced ROS production is attributed to $\mathrm{Ca}^{2+}$ binding to cardiolipin (51), to electromotive potentiation of superoxide formation at ubisemiquinone (25), and to $\mathrm{Ca}^{2+}$-sensitive mitochondrial dehydrogenases that augment the proton gradient by enhancing mitochondrial electron transport $(25,46)$. Whatever the mechanism, our data indicate that in lung capillary ECs, a twofold increase in the amplitude of $\mathrm{Ca}^{2+}$ cyt oscillations is sufficient to induce $\mathrm{Ca}^{2+}$ mit uptake and therefore mitochondrial ROS production. This attests to a sensitive $\mathrm{Ca}^{2+}$ uptake mechanism in these mitochondria.

Although the PEG-catalase experiments indicate that $\mathrm{H}_{2} \mathrm{O}_{2}$ was the major ROS responsible for P-selectin exocytosis, underlying mechanisms are unclear. One possibility is that ROS activate tyrosine kinases by inhibiting tyrosine phosphatases (52). ROS activate the Fyn tyrosine kinase as well as other Src family tyrosine kinases that are implicated in secretion $(53,54)$. The tyrosine residue on P-selectin has been previously implicated in the sorting of the receptor to secretory vesicles (55), suggesting a possible link between tyrosine phosphorylation and P-selectin exocytosis. These issues require further investigation in the context of our present findings.

Although mitochondria are understood to be the principal sources of cellular ATP, there is increasing recognition of a new mitochondrial role, namely as regulators of cell function. In the $\beta$ cell, mitochondria serve as fuel sensors, coupling nutrient metabolism to insulin secretion (56). In ECs, mitochondrial ROS induce expression of monocyte chemoattractant protein-1 (47). In lung, mitochondria act as oxygen sensors, generating ROS that induce hypoxic vasoconstriction (57). In T cells, mitochondria control $\mathrm{Ca}^{2+}$ channel activity and thereby regulate $\mathrm{T}$ cell activation (58). To this list, we may now add ECs of lung venular capillaries in which, as the present data show, mitochondria regulated the pressure-induced proinflammatory response. The mitochondrial role recognized here is that of organelles that actively induce ROS-induced signaling in response to the mechanical stress of vascular pressure elevation. The extent to which this mitochondrial role is a more general feature of the lung's response to other stress-inducing stimuli requires further consideration.

\section{Acknowledgments}

This work was supported by NIH grants HL-57556, HL-36024, and HL-64896 to J. Bhattacharya.

\footnotetext{
1. Kuebler, W.M., Parthasarathi, K., Wang, P.M., and Bhattacharya, J. 2000. A novel signaling mechanism between gas and blood compartments of the lung. J. Clin. Invest. 105:905-913.

2. Larsson, R., Rocksen, D., Lilliehook, B., Jonsson, A., and Bucht, A. 2000. Dose-dependent activation of lymphocytes in endotoxin-induced airway inflammation. Infect. Immun. 68:6962-6969.

3. Mizgerd, J.P., Spieker, M.R., and Doerschuk, C.M. 2001. Early response cytokines and innate immunity: essential roles for TNF receptor 1 and type I IL-1 receptor during Escherichia coli pneumonia in mice. J. Immunol. 166:4042-4048.

4. Kuebler, W.M., Ying, X., Singh, B., Issekutz, A.C., and Bhattacharya, J. 1999. Pressure is proinflammatory in lung venular capillaries. J. Clin. Invest. 104:495-502.

5. Bhattacharya, J. 1998. Physiological basis of pulmonary edema. In
} 
Pulmonary edema. M.A. Matthay and D.H. Ingbar, editors. Marcel Dekker Inc. New York, New York, USA. 1-36.

6. Ley, K., et al. 1995. Sequential contribution of L- and P-selectin to leukocyte rolling in vivo. J. Exp. Med. 181:669-675.

7. Mulligan, M.S., et al. 1992. Neutrophil-dependent acute lung injury. Requirement for P-selectin (GMP-140). J. Clin. Invest. 90:1600-1607.

8. Kyriakides, C., et al. 2000. Endothelial selectin blockade attenuates lung permeability of experimental acid aspiration. Surgery. 128:327-331.

9. Moore, T.M., Khimenko, P., Adkins, W.K., Miyasaka, M., and Taylor, A.E. 1995. Adhesion molecules contribute to ischemia and reperfusion-induced injury in the isolated rat lung. J. Appl. Physiol. 78:2245-2252.

10. Birch, K.A., Ewenstein, B.M., Golan, D.E., and Pober, J.S. 1994. Prolonged peak elevations in cytoplasmic free calcium ions, derived from intracellular stores, correlate with the extent of thrombin-stimulated exocytosis in single human umbilical vein endothelial cells. J. Cell. Physiol. 160:545-554.

11. Burgoyne, R.D., and Morgan, A. 1998. Calcium sensors in regulated exocytosis. Cell Calcium. 24:367-376.

12. Gunter, T.E., Buntinas, L., Sparagna, G., Eliseev, R., and Gunter, K. 2000. Mitochondrial calcium transport: mechanisms and functions. Cell Calcium. 28:285-296.

13. Kaftan, E.J., Xu, T., Abercrombie, R.F., and Hille, B. 2000. Mitochondria shape hormonally induced cytoplasmic calcium oscillations and modulate exocytosis. J. Biol. Chem. 275:25465-25470.

14. Kowaltowski, A.J., Naia-da-Silva, E.S., Castilho, R.F., and Vercesi, A.E. 1998. $\mathrm{Ca} 2+$-stimulated mitochondrial reactive oxygen species generation and permeability transition are inhibited by dibucaine or $\mathrm{Mg} 2+$. Arch. Biochem. Biophys. 359:77-81.

15. Chandel, N.S., Trzyna, W.C., McClintock, D.S., and Schumacker, P.T. 2000 Role of oxidants in NF-kappa B activation and TNF-alpha gene transcription induced by hypoxia and endotoxin. J. Immunol. 165:1013-1021.

16. Atlante, A., et al. 2000. Cytochrome $\mathrm{c}$ is released from mitochondria in a reactive oxygen species (ROS)-dependent fashion and can operate as a ROS scavenger and as a respiratory substrate in cerebellar neurons undergoing excitotoxic death. J. Biol. Chem. 275:37159-37166.

17. Boitier, E., Rea, R., and Duchen, M.R. 1999. Mitochondria exert a negative feedback on the propagation of intracellular $\mathrm{Ca} 2+$ waves in rat cortical astrocytes. J. Cell Biol. 145:795-808.

18. Ying, X., Minamiya, Y., Fu, C., and Bhattacharya, J. 1996. Ca2+ waves in lung capillary endothelium. Circ. Res. 79:898-908.

19. Wood, P.G., and Gillespie, J.I. 1998. Evidence for mitochondrial Ca(2+)induced $\mathrm{Ca} 2+$ release in permeabilised endothelial cells. Biochem. Biophys. Res. Commun. 246:543-548.

20. Madge, L., Marshall, I.C., and Taylor, C.W. 1997. Delayed autoregulation of the $\mathrm{Ca} 2+$ signals resulting from capacitative $\mathrm{Ca} 2+$ entry in bovine pulmonary artery endothelial cells. J. Physiol. 498:351-369.

21. Trollinger, D.R., Cascio, W.E., and Lemasters, J.J. 2000. Mitochondrial calcium transients in adult rabbit cardiac myocytes: inhibition by ruthenium red and artifacts caused by lysosomal loading of $\mathrm{Ca}(2+)$-indicating fluorophores. Biophys. J. 79:39-50.

22. Collins, T.J., Lipp, P., Berridge, M.J., and Bootman, M.D. 2001. Mitochondrial $\mathrm{Ca}(2+)$ uptake depends on the spatial and temporal profile of cytosolic $\mathrm{Ca}(2+)$ signals. J. Biol. Chem. 276:26411-26420.

23. Sanchez,J.A., et al. 2001. Mitochondria regulate inactivation of L-type Ca2+ channels in rat heart. J. Physiol. 536:387-396.

24. Petronilli, V., Penzo, D., Scorrano, L., Bernardi, P., and Di Lisa, F. 2001. The mitochondrial permeability transition, release of cytochrome $\mathrm{c}$ and cell death. Correlation with the duration of pore openings in situ. J. Biol. Chem. 276:12030-12034.

25. Korshunov, S.S., Skulachev, V.P., and Starkov, A.A. 1997. High protonic potential actuates a mechanism of production of reactive oxygen species in mitochondria. FEBS Lett. 416:15-18.

26. Gafni, J., et al. 1997. Xestospongins: potent membrane permeable blockers of the inositol 1,4,5-trisphosphate receptor. Neuron. 19:723-733.

27. Thastrup, O., Cullen, P.J., Drobak, B.K., Hanley, M.R., and Dawson, A.P. 1990. Thapsigargin, a tumor promoter, discharges intracellular $\mathrm{Ca} 2+$ stores by specific inhibition of the endoplasmic reticulum Ca2(+)-ATPase. Proc. Natl. Acad. Sci. U. S. A. 87:2466-2470.

28. Parthasarathi, K., Ichimura, H., Quadri, S., Issekutz, A., and Bhattacharya, J. 2002. Mitochondrial reactive oxygen species regulate spatial profile of proinflammatory responses in lung venular capillaries. J. Immunol. 169:7078-7086.

29. Rosenkranz, A.R., et al. 1992. A microplate assay for the detection of oxidative products using 2',7'-dichlorofluorescin-diacetate. J. Immunol. Methods. 156:39-45.

30. Scheffler, I.E. 1999. Mitochondrial electron transport and oxidative phosphorylation. In Mitochondria. I.E. Scheffler, editor. John Wiley \& Sons. New York, New York, USA. 141-245.

31. Burow, S., and Valet, G. 1987. Flow-cytometric characterization of stimulation, free radical formation, peroxidase activity and phagocytosis of human granulocytes with 2,7-dichlorofluorescein (DCF). Eur. J. Cell Biol. 43:128-133.
32. Rota, C., Fann, Y.C., and Mason, R.P. 1999. Phenoxyl free radical formation during the oxidation of the fluorescent dye 2',7'-dichlorofluorescein by horseradish peroxidase. Possible consequences for oxidative stress measurements. J. Biol. Chem. 274:28161-28168.

33. Royall, J.A., and Ischiropoulos, H. 1993. Evaluation of $2^{\prime}, 7^{\prime}$-dichlorofluorescin and dihydrorhodamine 123 as fluorescent probes for intracellular $\mathrm{H} 2 \mathrm{O} 2$ in cultured endothelial cells. Arch. Biochem. Biophys. 302:348-355.

34. Imrich, A., and Kobzik, L. 1997. Fluorescence-based measurement of nitric oxide synthase activity in activated rat macrophages using dichlorofluorescin. Nitric Oxide. 1:359-369.

35. Kojima, H., et al. 1998. Detection and imaging of nitric oxide with novel fluorescent indicators: diaminofluoresceins. Anal. Chem. 70:2446-2453.

36. Broillet, M., Randin, O., and Chatton, J. 2001. Photoactivation and calcium sensitivity of the fluorescent $\mathrm{NO}$ indicator 4,5-diaminofluorescein (DAF-2): implications for cellular NO imaging. FEBS Lett. 491:227-232.

37. Bove, K., Neumann, P., Gertzberg, N., and Johnson, A. 2001. Role of ecNOS-derived NO in mediating TNF-induced endothelial barrier dysfunction. Am. J. Physiol. Lung Cell. Mol. Physiol. 280:L914-L922.

38. Davis, S., Weiss, M.J., Wong, J.R., Lampidis, T.J., and Chen, L.B. 1985. Mitochondrial and plasma membrane potentials cause unusual accumulation and retention of rhodamine 123 by human breast adenocarcinomaderived MCF-7 cells. J. Biol. Chem. 260:13844-13850.

39. Floryk, D., and Houstek, J. 1999. Tetramethyl rhodamine methyl ester (TMRM) is suitable for cytofluorometric measurements of mitochondrial membrane potential in cells treated with digitonin. Biosci. Rep. 19:27-34.

40. Hu, Q., and Ziegelstein, R.C. 2000. Hypoxia/reoxygenation stimulates intracellular calcium oscillations in human aortic endothelial cells. Circulation. 102:2541-2547.

41. Howard, A.B., Alexander, R.W., Nerem, R.M., Griendling, K.K., and Taylor, W.R. 1997. Cyclic strain induces an oxidative stress in endothelial cells. Am. J. Physiol. 272:C421-C427.

42. Hishikawa, K., and Luscher, T.F. 1997. Pulsatile stretch stimulates superoxide production in human aortic endothelial cells. Circulation. 96:3610-3616.

43. Wung, B.S., Cheng, J.J., Shyue, S.K., and Wang, D.L. 2001. NO modulates monocyte chemotactic protein-1 expression in endothelial cells under cyclic strain. Arterioscler. Thromb. Vasc. Biol. 21:1941-1947.

44. Carriedo, S.G., Sensi, S.L., Yin, H.Z., and Weiss, J.H. 2000. AMPA exposures induce mitochondrial $\mathrm{Ca}(2+)$ overload and ROS generation in spinal motor neurons in vitro. J. Neurosci. 20:240-250.

45. Fernandez-Chacon, R., et al. 2001. Synaptotagmin I functions as a calcium regulator of release probability. Nature. 410:41-49.

46. Hajnoczky, G., Robb-Gaspers, L.D., Seitz, M.B., and Thomas, A.P. 1995 Decoding of cytosolic calcium oscillations in the mitochondria. Cell. 82:415-424.

47. Yamagishi, S.I., et al. 2001. Leptin induces mitochondrial superoxide production and monocyte chemoattractant protein-1 expression in aortic endothelial cells by increasing fatty acid oxidation via protein kinase A J. Biol. Chem. 276:25096-25100.

48. Reynolds, I.J., and Hastings, T.G. 1995. Glutamate induces the production of reactive oxygen species in cultured forebrain neurons following NMDA receptor activation. J. Neurosci. 15:3318-3327.

49. Collins, T.J., Lipp, P., Berridge, M.J., Li, W., and Bootman, M.D. 2000. Inositol 1,4,5-trisphosphate-induced $\mathrm{Ca} 2+$ release is inhibited by mitochondrial depolarization. Biochem. J. 347:593-600.

50. Sengpiel, B., Preis, E., Krieglstein, J., and Prehn, J.H. 1998. NMDA-induced superoxide production and neurotoxicity in cultured rat hippocampal neurons: role of mitochondria. Eur. J. Neurosci. 10:1903-1910.

51. Grijalba, M.T., Vercesi, A.E., and Schreier, S. 1999. Ca2+-induced increased lipid packing and domain formation in submitochondrial particles. A possible early step in the mechanism of $\mathrm{Ca} 2+$-stimulated generation of reactive oxygen species by the respiratory chain. Biochemistry. 38:13279-13287.

52. Sattler, M., et al. 2000. The BCR/ABL tyrosine kinase induces production of reactive oxygen species in hematopoietic cells. J. Biol. Chem. 275:24273-24278

53. Allen, C.M., Ely, C.M., Juaneza, M.A., and Parsons, S.J. 1996. Activation of Fyn tyrosine kinase upon secretagogue stimulation of bovine chromaffin cells. J. Neurosci. Res. 44:421-429.

54. Mocsai, A., et al. 2000. Kinase pathways in chemoattractant-induced degranulation of neutrophils: the role of $\mathrm{p} 38$ mitogen-activated protein kinase activated by Src family kinases. J. Immunol. 164:4321-4331.

55. Hartwell, D.W., et al. 1998. Role of P-selectin cytoplasmic domain in granular targeting in vivo and in early inflammatory responses. J. Cell Biol. 143:1129-1141.

56. Maechler, P., and Wollheim, C.B. 1999. Mitochondrial glutamate acts as a messenger in glucose-induced insulin exocytosis. Nature. 402:685-689.

57. Chandel, N.S., et al. 2000. Reactive oxygen species generated at mitochondrial complex III stabilize hypoxia-inducible factor-1alpha during hypoxia: a mechanism of O2 sensing. J. Biol. Chem. 275:25130-25138.

58. Hoth, M., Fanger, C.M., and Lewis, R.S. 1997. Mitochondrial regulation of store-operated calcium signaling in T lymphocytes. J. Cell Biol. 137:633-648. 